\title{
Analysis of aqueductal cerebrospinal fluid flow after endoscopic aqueductoplasty by using cine phase-contrast magnetic resonance imaging
}

\author{
Henry W. S. Schroeder, M.D., Christiane Schweim, Klaus H. Schweim, M.D., \\ and Michael R. GaAb, M.D., Ph.D.
}

Department of Neurosurgery, Ernst Moritz Arndt University, Greifswald, Germany; and Department of Radiology and Radiological Practice, Stralsund, Germany

\begin{abstract}
Object. The purpose of this prospective study was to evaluate aqueductal cerebrospinal fluid (CSF) flow after endoscopic aqueductoplasty. In all patients, preoperative magnetic resonance (MR) imaging revealed hydrocephalus caused by aqueductal stenosis and lack of aqueductal CSF flow.

Methods. In 14 healthy volunteers and in eight patients with aqueductal stenosis who had undergone endoscopic aqueductoplasty, aqueductal CSF flow was investigated using cine cardiac-gated phase-contrast MR imaging. For qualitative evaluation of CSF flow, the authors used an in-plane phase-contrast sequence in the midsagittal plane. The MR images were displayed in a closed-loop cine format. Quantitative through-plane measurements were performed in the axial plane perpendicular to the aqueduct. Evaluation revealed no significant difference in aqueductal CSF flow between healthy volunteers and patients with regard to temporal parameters, CSF peak and mean velocities, mean flow, and stroke volume. All restored aqueducts have remained patent 7 to 31 months after surgery.

Conclusions. Aqueductal CSF flow after endoscopic aqueductoplasty is similar to aqueductal CSF flow in healthy volunteers. The data indicate that endoscopic aqueductoplasty seems to restore physiological aqueductal CSF flow.
\end{abstract}

KEY WORDS - endoscopic aqueductoplasty •

cine phase-contrast magnetic resonance imaging - cerebrospinal fluid flow • cerebrospinal fluid circulation • aqueduct

$\mathrm{C}$ INE phase-contrast MR imaging has been increasingly used during the last decade for evaluating cranial and spinal CSF flow. $4,7,11,17,18,39,49$ The phasecontrast technique is extremely sensitive, even to slow flow, and provides the potential for noninvasive flow quantification. , $17,28,60$ The results of these measurements have yielded considerable information on the physiology of the normal CSF circulation. $1,6,7,9,17,23,42,43$ In addition, pathological CSF flow dynamics in communicating and obstructive hydrocephalus, ${ }^{11,22,32-34,44,54}$ Chiari malformation, ${ }^{8}$ cystic lesion, ${ }^{15}$ and cervical spondylosis ${ }^{60}$ have been analyzed. With the increasing frequency of neuroendoscopic procedures, cine MR imaging has been recommended for evaluating the patency of third ventriculostomies. ${ }^{21,37,38,40,48}$ Aqueductal CSF flow after endoscopic aqueductoplasty, however, has not yet been investigated. We performed a prospective study to compare the aqueductal CSF flow in healthy volunteers and patients who had undergone endoscopic restoration of aqueductal patency.

Abbreviations used in this paper: $\mathrm{CSF}=$ cerebrospinal fluid; $\mathrm{MR}=$ magnetic resonance; $\mathrm{ROI}=$ region of interest.

\section{Clinical Material and Methods}

\section{Patients and Healthy Volunteers}

Fourteen healthy volunteers and eight patients who underwent endoscopic aqueductoplasty were prospectively investigated using cine phase-contrast MR imaging to evaluate aqueductal CSF flow. All patients had occlusive hydrocephalus caused by aqueductal stenosis and presented with symptoms of increased intracranial pressure or normal-pressure hydrocephalus before surgery. Flow-sensitive sagittal $\mathrm{T}_{2}$-weighted turbo inversion-recovery spinecho MR imaging revealed the lack of an aqueductal flow-void sign in all cases. The operative technique of endoscopic aqueductoplasty has been reported elsewhere. ${ }^{50}$ In brief, the aqueduct was approached via a frontal paramedian burr hole. Endoscopic inspection revealed three membranous obstructions, two membranous stenoses with minimal residual lumina, and three short stenoses approximately $2 \mathrm{~mm}$ in length. The membranous stenoses were perforated using a steerable $2.5-\mathrm{mm}$ fiberscope. The short stenoses were dilated by inflating the balloon of a No. 3 French Fogarty catheter. The healthy control volunteers exhibited no clinical or radiological signs of CSF circu- 
H. W. S. Schroeder, et al.

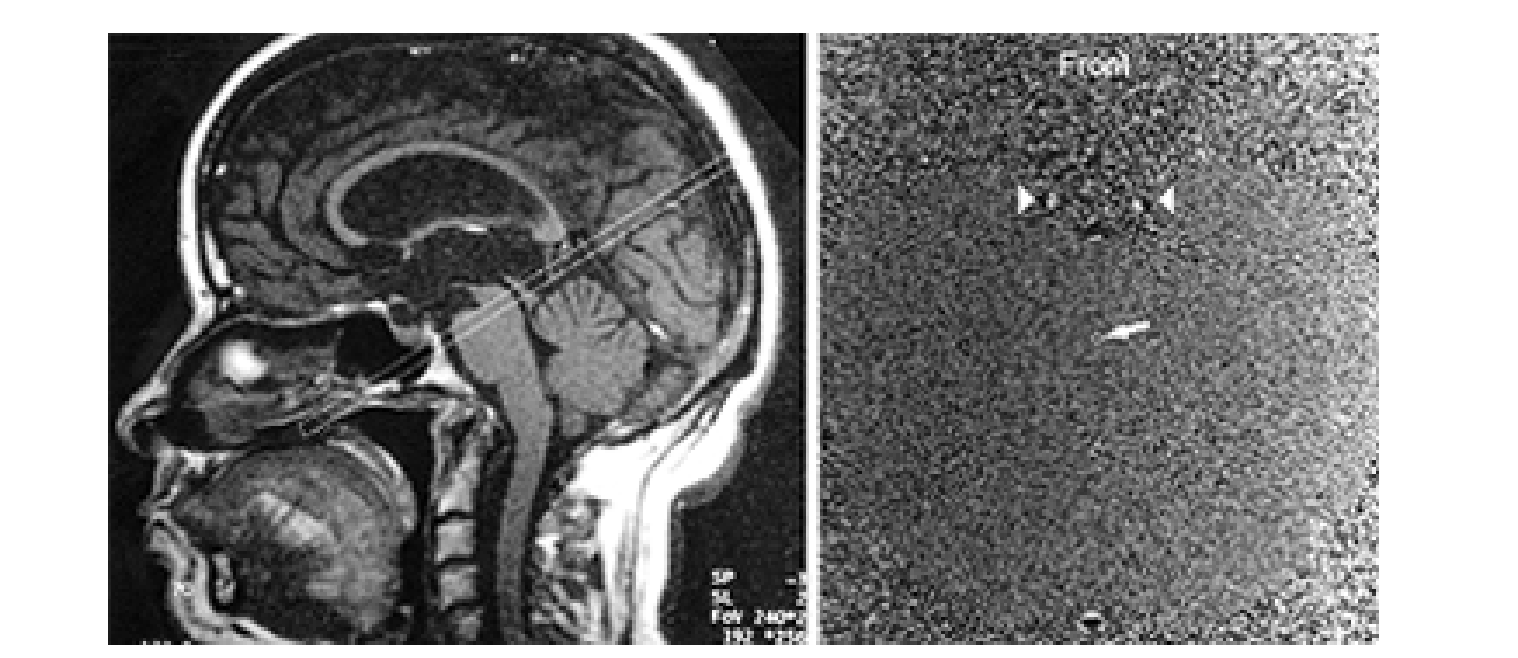

FIG. 1. Quantification of CSF flow. Left: Sagittal $\mathrm{T}_{1}$-weighted scout MR image demonstrating the phase-contrast imaging plane perpendicular to the aqueduct (dotted bar) used to assess aqueductal CSF flow. Right: Axial phase-contrast image perpendicular to the aqueduct, demonstrating an annular ROI (arrow) located within the aqueduct. Arrowheads indicate the internal carotid arteries.

lation abnormalities. Both patients (five men and three women) and volunteers (six men and eight women) were normotensive. Their heart rates varied between 55 and 85 beats/minute. The mean age of the patients was 40 years (range 18-56 years) and the mean age of the volunteers was 38 years (range $20-66$ years).

\section{Magnetic Resonance Imaging Techniques}

Magnetic resonance imaging was performed using a 1.5-tesla MR imaging unit (Magnetom Symphony; Siemens AG, Erlangen, Germany) with a circular polarized head-array coil and ultra gradients. Standard sagittal (2$\mathrm{mm}$ slice thickness) and axial (5-mm slice thickness) $\mathrm{T}_{1}{ }^{-}$

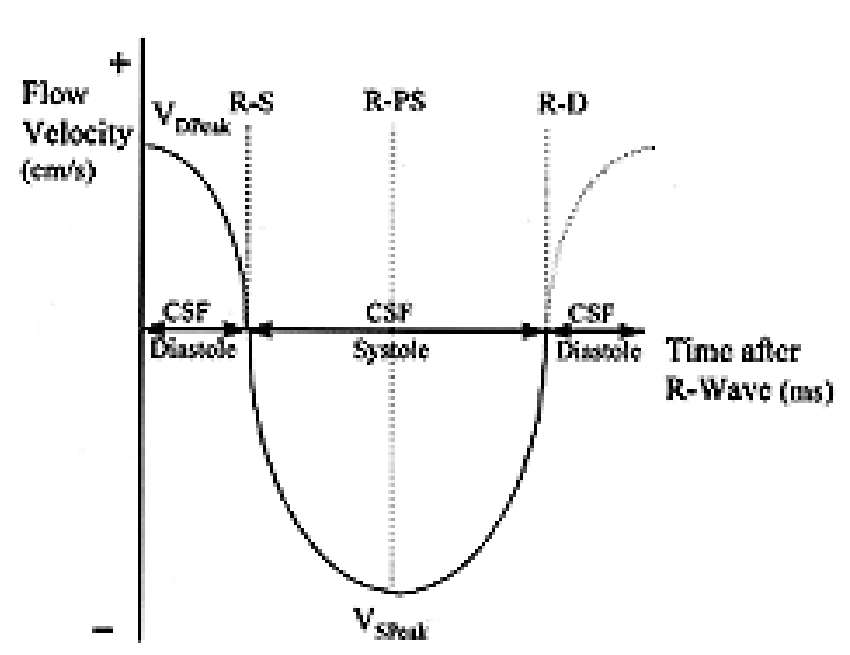

FIG. 2. Graph depicting the CSF flow waveform. Positive deflections represent caudocranial flow (CSF diastole) and negative deflection represents craniocaudal flow (CSF systole). Dotted lines indicate a portion of the waveform that was not evaluated because of prospective cardiac gating. $\mathrm{R}-\mathrm{D}=$ time after $\mathrm{R}$ wave to onset of CSF diastole; R-PS = time after R wave to peak CSF systole; $\mathrm{R}-\mathrm{S}=$ time after $\mathrm{R}$ wave to onset of CSF systole; $\mathrm{V}_{\text {Deak }}=$ peak enddiastolic CSF velocity; $\mathrm{V}_{\text {SPeak }}=$ peak systolic CSF velocity. weighted images, as well as sagittal $\mathrm{T}_{2}$-weighted turbo inversion-recovery spin-echo images (TE $4300 \mathrm{msec}$, TE 60 msec, and 2-mm slice thickness), were obtained before CSF flow measurements were made. For phase-contrast MR imaging, we used a two-dimensional fast low-angle shot sequence from a commercially available flow quantification package (included in the Numaris VA11B software, version 3.5; Siemens AG). Two imaging techniques were applied: one in the axial plane with through-plane velocity encoding in the craniocaudal direction for flow quantification, and one in the sagittal plane with in-plane velocity encoding in the craniocaudal direction for qualitative assessment. ${ }^{46}$

\section{Axial Technique}

Cerebrospinal fluid flow dynamics were quantitatively studied by using a prospectively cardiac-gated high-resolution axial phase-contrast protocol with an imaging plane perpendicular to the aqueduct (Fig. 1 left). The direction of flow encoding was craniocaudal. The imaging parameters were as follows: TR $46 \mathrm{msec}$; TE $11 \mathrm{msec}$; flip angle $10^{\circ}$; number of acquisitions 2; field of view $160 \mathrm{~mm}$; matrix $256 \times 512$; pixel size $0.63 \times 0.31 \mathrm{~mm}$; scan thickness $4 \mathrm{~mm}$; and velocity encoding $20 \mathrm{~cm} / \mathrm{second}$. Depending on the patient's heart rate, the measurement time lasted between 10 to 15 minutes, and 14 to 23 phase images were calculated. Once the image data had been acquired, an annular ROI was placed in the aqueduct shown on a magnified image (Fig. 1 right) with the aid of a mouse-driven cursor, and a CSF flow waveform was generated (Fig. 2). The time of the cardiac cycle after the $\mathrm{R}$ wave was plotted on the $\mathrm{x}$ axis and the velocity on the $\mathrm{y}$ axis. During CSF diastole, CSF moves in caudocranial direction (positive velocity), whereas during CSF systole, CSF flows in the craniocaudal direction (negative velocity). Mean and peak CSF velocities, as well as mean CSF flow, were calculated at the end of CSF diastole and during CSF systole ( $\mathrm{sol}$ id line in Fig. 2). The early diastole (curved dotted line on right side of Fig. 2) was not evaluated because, in prospec- 


\section{Cerebrospinal fluid flow after endoscopic aqueductoplasty}

tive cardiac gating, the acquisition is stopped within approximately 150 to $200 \mathrm{msec}$ of the next R wave for accurate detection of the next trigger. Hence, the entire diastolic phase is not covered. ${ }^{46}$ Temporal analysis involved determination of the interval from the $\mathrm{R}$ wave to the onset of CSF systole (R-S), the interval from the $\mathrm{R}$ wave to the peak CSF systole (R-PS), and the interval from the $\mathrm{R}$ wave to the onset of CSF diastole (R-D). In addition, duration of the systole and systolic CSF stroke volume were calculated and the aqueductal area measured.

\section{Sagittal Technique}

For qualitative assessment of CSF flow, midsagittal phase-contrast images were displayed in a closed-loop cine format. Retrospective cardiac gating was used to cover the whole cardiac cycle. ${ }^{46}$ Thirty-two phase images were calculated. The direction of flow encoding was craniocaudal. The imaging parameters were as follows: TR $70 \mathrm{msec}$; TE $18 \mathrm{msec}$; flip angle $15^{\circ}$; number of acquisitions 1; field of view $250 \mathrm{~mm}$; matrix $193 \times 256$; pixel size $1.13 \times 0.98 \mathrm{~mm}$; scan thickness $4 \mathrm{~mm}$; and velocity encoding $2 \mathrm{~cm} / \mathrm{second}$. Measurement time was approximately 7 minutes.

\section{Statistical Analysis}

Statistical evaluation was performed using a commercially available computer software package (SAS System for Windows, version 6.12; SAS Institute, Inc., Cary, NC). The range, mean, and standard deviation were calculated for each parameter. The Wilcoxon rank-sum test was used for a comparison of parameters in healthy volunteers and patients. Probability values less than 0.05 were considered significant.

\section{Results}

\section{Routine MR Imaging}

Standard $\mathrm{T}_{1}$-weighted images revealed restored aqueducts in all patients (Fig. 3 and Video Clip 1). There was a decrease in the size of the ventricles and reversal of the distortion of the pituitary stalk in six patients. In two patients, ventricular size remained unchanged. Flow-sensitive sagittal inversion-recovery images demonstrated an

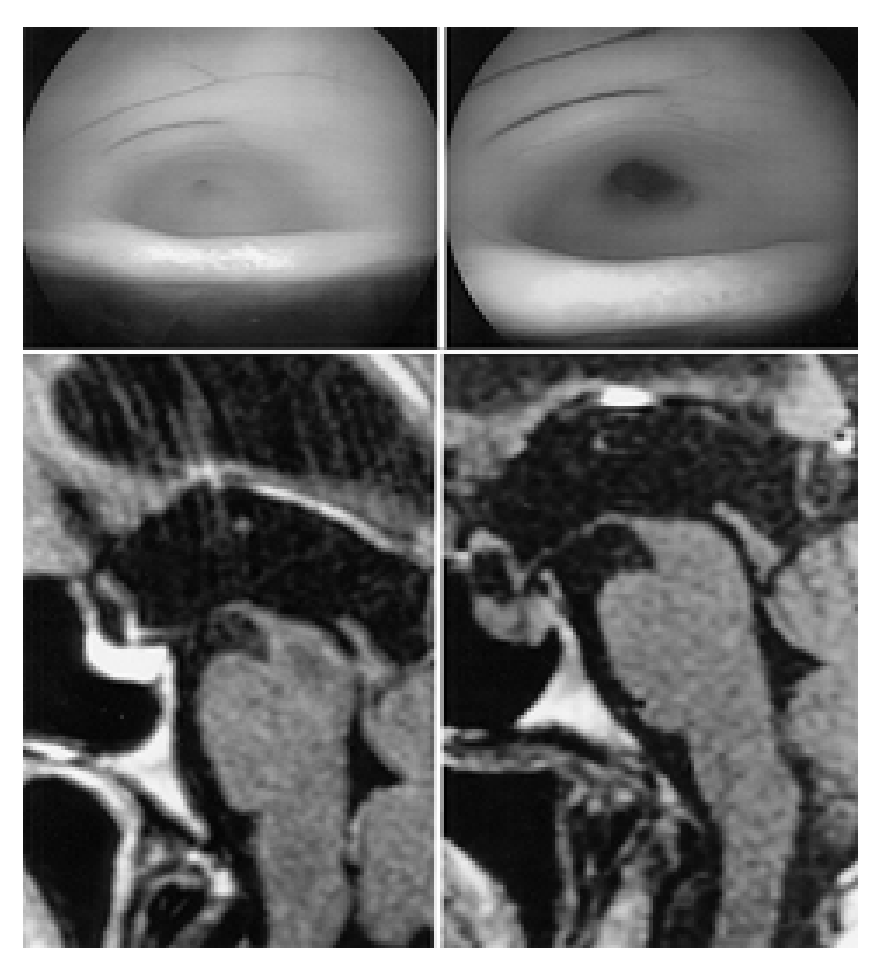

FIG. 3. Upper Left and Right: Endoscopic views showing aqueductal stenosis with a minimal residual lumen (left) and restored aqueduct after aqueductoplasty (right). Lower Left: Preoperative sagittal $T_{1}$-weighted MR image revealing a short aqueduct stenosis and a dilated third ventricle. Note the wide angle of infundibular recess. Lower Right: Sagittal $\mathrm{T}_{1}$-weighted MR image obtained 20 months after surgery demonstrating a normal-shaped aqueduct and a slightly decreased third ventricle. Note the small angle of infundibular recess. See Video Clip 1.

aqueductal flow-void sign in all patients. However, in one patient in whom there was a very narrow aqueduct $(<1$ $\mathrm{mm}^{2}$ ) the flow-void sign was very weak. Magnetic resonance imaging of healthy volunteers revealed normalshaped aqueducts and ventricles and no signs of CSF circulation abnormalities. In two volunteers with narrow aqueducts $\left(<1 \mathrm{~mm}^{2}\right)$, a flow-void sign was hardly detectable.

TABLE 1

Quantitative assessment of aqueductal CSF flow in 14 healthy volunteers and eight patients who had undergone endoscopic aqueductoplasty

\begin{tabular}{|c|c|c|c|c|}
\hline \multirow[b]{2}{*}{ Parameter } & \multicolumn{2}{|c|}{ Range } & \multicolumn{2}{|c|}{ Mean \pm Standard Deviation } \\
\hline & Healthy Volunteers & Patients & Healthy Volunteers & Patients \\
\hline end-diastolic mean velocity $(\mathrm{cm} / \mathrm{sec})$ & $1.95-6.54$ & $1.24-5.11$ & $3.48 \pm 1.22$ & $2.94 \pm 1.43$ \\
\hline systolic peak velocity $(\mathrm{cm} / \mathrm{sec})$ & $2.59-8.63$ & $1.5-8.16$ & $5.56 \pm 1.87$ & $4.95 \pm 1.98$ \\
\hline systolic mean velocity $(\mathrm{cm} / \mathrm{sec})$ & $1.61-4.80$ & $0.76-4.60$ & $3.07 \pm 1.09$ & $2.96 \pm 1.37$ \\
\hline onset of CSF systole (R-S; msec) & $139-196$ & $123-227$ & $167 \pm 18$ & $159 \pm 31$ \\
\hline time of CSF peak systole (R-PS; msec) & $232-414$ & $233-411$ & $326 \pm 51$ & $299 \pm 59$ \\
\hline duration of CSF systole (msec) & $303-586$ & $354-555$ & $465 \pm 84$ & $441 \pm 65$ \\
\hline systolic stroke volume $(\mu \mathrm{l})$ & 4-115 & $4-57$ & $28 \pm 28.4$ & $24 \pm 16.6$ \\
\hline aqueductal area $\left(\mathrm{mm}^{2}\right)$ & $<1-5$ & $<1-5$ & $1.7 \pm 1.4$ & $1.8 \pm 1.6$ \\
\hline
\end{tabular}


H. W. S. Schroeder, et al.

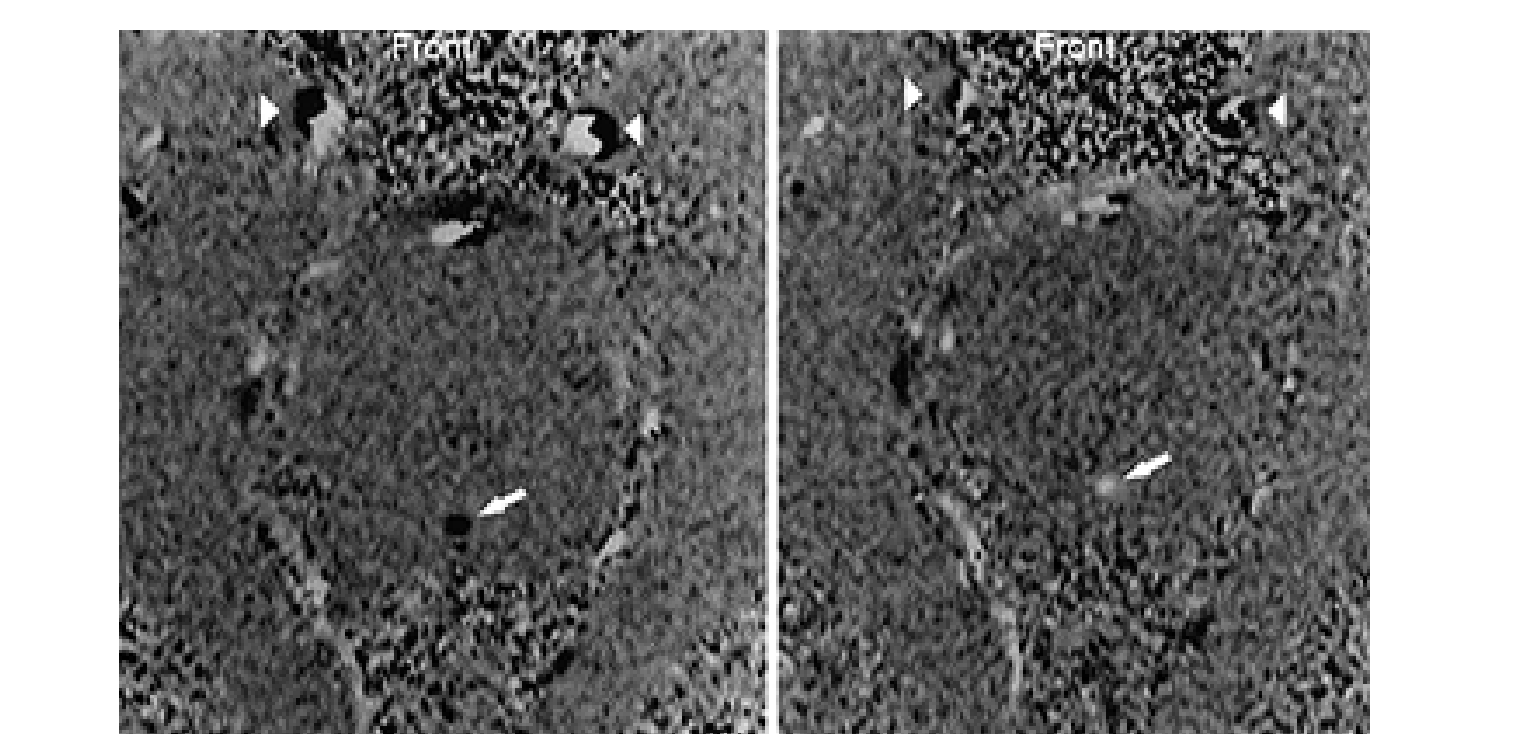

FIG. 4. Axial phase-contrast images obtained perpendicular to the aqueduct. Arrowheads indicate the carotid arteries. Left: Hypointense signal within the aqueduct (arrow) indicating cranial flow (CSF diastole). Right: Hyperintense signal within the aqueduct (arrow) indicating caudal flow (CSF systole). See Video Clip 2.

Video Clip 1: [6.2 MB] Endoscopic aqueductoplasty. The endoscope is introduced into the posterior part of the third ventricle via the foramen of Monro. Endoscopic inspection reveals a short aqueductal stenosis with a minimal residual lumen. A No. 3 French Fogarty catheter (note the bending of the catheter tip) is carefully inserted into the stenotic aqueduct, and the stenosis is dilated by inflating the balloon. Final inspection with the diagnostic endoscope shows the restored aqueduct.

\section{Quantitative Assessment}

An overview of the measured and calculated parameters of aqueductal CSF flow are presented in Table 1. Axial phase-contrast images revealed aqueductal cranial flow during CSF diastole (hypointense signal) and caudal flow during CSF systole (hyperintense signal; Fig. 4 and Video Clip 2). The CSF waveforms generated were similar in patients and healthy volunteers (Fig. 5). There were no significant differences between patients and healthy volunteers regarding aqueductal area, velocity, flow, and temporal parameters. The high variability of end-diastolic $(1.74-9.26 \mathrm{~cm} / \mathrm{second})$ and systolic $(1.5-8.63 \mathrm{~cm} / \mathrm{second})$ peak CSF velocities in both patients and healthy volunteers is striking. Mean CSF flows at the end of CSF diastole (0.06-0.07 ml/second) and during CSF systole (0.06 $\mathrm{ml} /$ second) were nearly equivalent in patients and volunteers. Caudal flow (CSF systole) started approximately $165 \mathrm{msec}$ after the R wave and lasted approximately 450 msec. The calculated volume of CSF that flows caudally through the aqueduct during CSF systole was $24 \mu \mathrm{l}$ in patients and $28 \mu \mathrm{l}$ in healthy volunteers. The aqueductal area varied from less than 1 to $5 \mathrm{~mm}^{2}$ in both patients and Video Clip 2: [4 MB] fine MR imaging CSF flow quantilication. For quantitative assessment (velocity and flow measurements), cine MR imaging is performed in an imaging plane perpendicular to the aqueduct with through-plane velocity encoding in the craniocaudal di- rection. Cerebrospinal fluid flow dynamics are studied by using a prospectively cardiac-gated high-resolution axial phase-contrast protocol. The imaging parameters are as follows: TR $46 \mathrm{msec}$; TE $11 \mathrm{msec}$; flip angle $10^{\circ}$; number of acquisitions 2; field of view $160 \mathrm{~mm}$; matrix $256 \times$ 512; pixel size $0.63 \mathrm{~mm} \times 0.31 \mathrm{~mm}$; scan thickness 4 $\mathrm{mm}$; velocity encoding $20 \mathrm{~cm} / \mathrm{second}$. Depending on the heart rate, measurement time is between 10 and 15 minutes and 14 to 23 phase images are calculated. The early diastole is not evaluated, because in prospective cardiac gating the acquisition is stopped within approximately 150 to $200 \mathrm{msec}$ of the next $\mathrm{R}$ wave for accurate detection of the next trigger. Therefore, the entire diastolic phase is not covered. The phase-contrast images are displayed in a closed cine loop format. Aqueductal cranial flow in CSF diastole is indicated as a hypointense signal and caudal flow in CSF systole as a hyperintense signal.

\section{Qualitative Assessment}

Sagittal phase-contrast images revealed aqueductal CSF flow in all patients and healthy volunteers. However, in one volunteer with a narrow aqueduct (aqueduct area $<$ $1 \mathrm{~mm}^{2}$ ), the sign of flow was weak. Caudal CSF flow during CSF systole was indicated as a hypointense signal, whereas cranial flow during CSF diastole was shown as a hyperintense signal (Fig. 6 and Video Clip 3). The general CSF flow pattern was similar in patients and healthy volunteers. At the end of CSF diastole, immediately after the R wave, CSF flow in the upper cervical subarachnoid space, prepontine cistern, and aqueduct was directed cranially (Fig. 6A). Ce-rebrospinal fluid systole started in the upper cervical subarachnoid space and preceded caudal flow in the prepontine cistern and aqueduct (Fig. 6B). During midsystole, CSF motion proceeded in a caudal direction in all CSF pathways (Fig. 6C). Onset of CSF diastole occurred first in the upper cervical subarachnoid space followed by flow reversal in the prepontine cistern and aqueduct. During the middle of diastole, flow again 
was directed cranially in the cervical subarachnoid space, prepontine cistern, and aqueduct (Fig. 6D).

video Clip 3. [5.2 NH]Cine MR imaging CSF flow visuatization. For qualitative assessment (flow visualization), cine MR imaging is performed in the midsagittal plane with in-plane velocity encoding in the craniocaudal direction. Retrospective cardiac gating is used to cover the whole cardiac cycle. Thirty-two phase images are calculated. The imaging parameters are as follows: TR 70 msec; TE $18 \mathrm{msec}$; flip angle $15^{\circ}$; number of aquisitions 1; field of view $250 \mathrm{~mm}$; matrix $193 \times 256$; pixel size $1.13 \mathrm{~mm} \times 0.98 \mathrm{~mm}$; scan thickness $4 \mathrm{~mm}$; velocity encoding $2 \mathrm{~cm} / \mathrm{second}$. Measurement time is approximately 7 minutes. The phase-contrast images are displayed in a continuously repeating cine loop format. Caudal CSF flow in CSF systole is indicated as a hypointense signal, whereas cranial flow during CSF diastole is shown as a hyperintense signal. In CSF end diastole immediately after the $\mathrm{R}$ wave, CSF flow in the upper cervical subarachnoid space, prepontine cistern, and aqueduct is directed cranially. Cerebrospinal fluid systole started in the upper cervical subarachnoid space and preceded caudal flow in the prepontine cistern and aqueduct. In late systole, CSF motion is in caudal direction in all CSF pathways. In mid diastole, flow is again directed cranially in cervical subarachnoid space, prepontine cistern, and aqueduct.

\section{Discussion}

Magnetic resonance imaging has provided considerable information regarding CSF dynamics. ${ }^{4,42,43}$ Initially, CSF flow was only qualitatively described as the flow-void sign that is best appreciated in areas of narrowing within the ventricular system such as the aqueduct. ${ }^{2,10,53-55,62}$ The visualization of this effect on routine $T_{1}$ - or $T_{2}$-weighted MR images is not consistent. However, on sagittal $\mathrm{T}_{2^{-}}$ weighted turbo inversion-recovery MR images, an aqueductal flow-void sign can be detected in most healthy volunteers. If the aqueduct is very narrow (aqueduct area $<$ $1 \mathrm{~mm}^{2}$ ), the sign may be very weak or even absent. In all patients in this series, a flow-void sign indicating patency of the restored aqueduct could be detected. For routine follow-up examination after endoscopic third ventriculostomy or aqueductoplasty, we use this turbo inversion recovery sequence. If no flow void can be seen, cine phasecontrast MR imaging is performed.

During the last decade, flow-sensitive cardiac-gated phase-contrast MR imaging techniques have been increasingly applied to study CSF flow dynamics both qualitatively and quantitatively., ${ }^{9,11,14,18,46}$ The results of our study support the current opinion on physiological CSF circulation. Early during the cardiac cycle, immediately after the $\mathrm{R}$ wave, there is still CSF motion in the caudocranial direction, which is referred to as CSF diastole in all CSF spaces. The CSF systole (motion in the craniocaudal direction) starts in the upper cervical subarachnoid space and then in the basal cisterns. Systolic flow within ventricles and aqueduct is slightly delayed compared with that of the cisterns. The CSF diastole starts in the upper cervical subarachnoid space and then in the basal cisterns, followed by the aqueduct and ventricles. . $^{78,24,46,49}$

Cerebrospinal fluid flow within the aqueduct is best

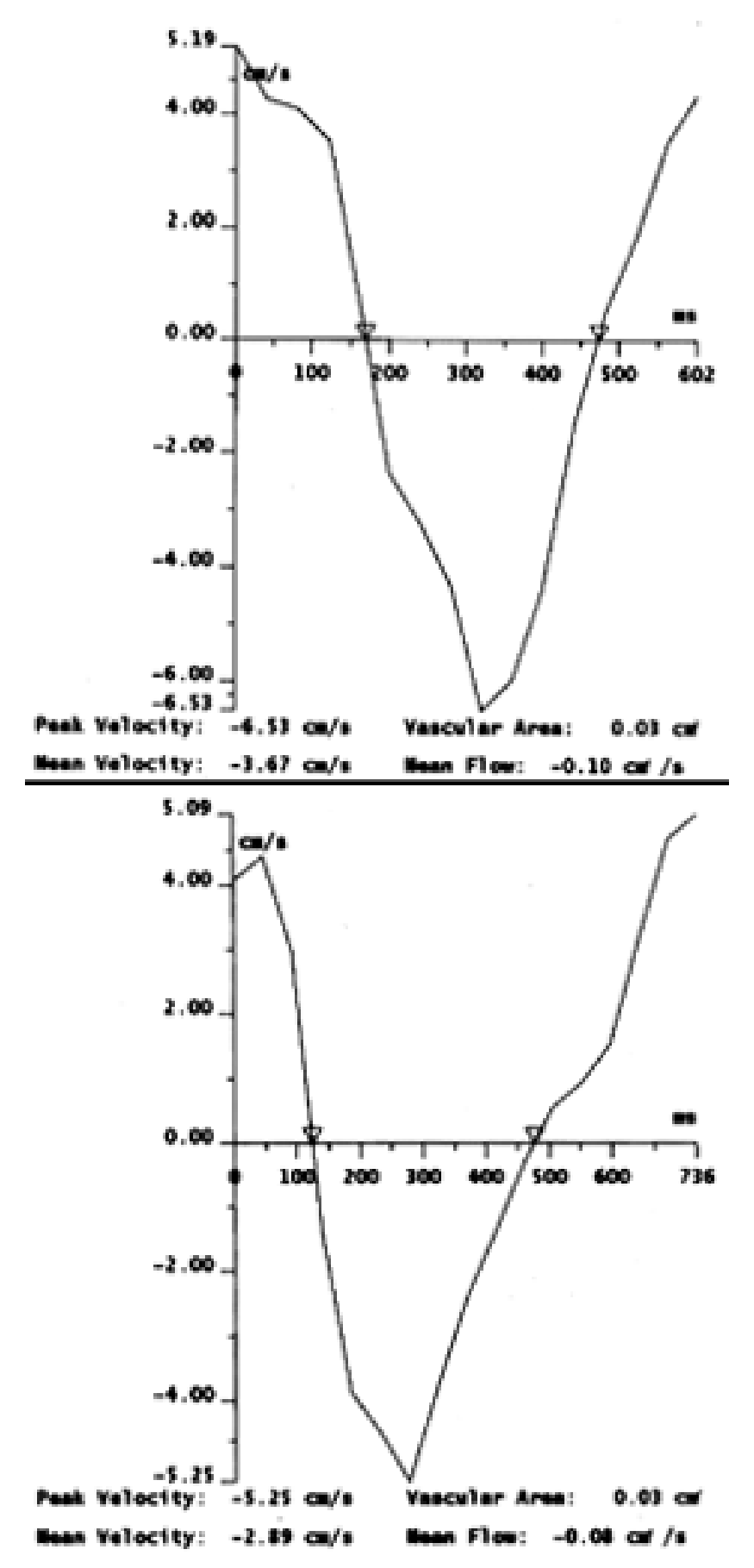

FIG. 5. Graphs showing CSF velocity profiles in a healthy volunteer (upper) and a patient 20 months after aqueductoplasty (lower). (Data were obtained in the same patient represented in Figs. 3 and 4).

described as a to-and-fro motion with a very small net flow. ${ }^{49}$ The wide physiological range of the temporal, velocity, and flow parameters is striking. ${ }^{6,7,28,34,59}$ This normal variation is mainly related to the size and anatomy of CSF spaces, size of blood vessels, systolic and diastolic arterial blood pressure, heart rate, jugular venous flow, compliance of surrounding brain tissue, ${ }^{1,6,12,49}$ and respiration..$^{41,52}$ 


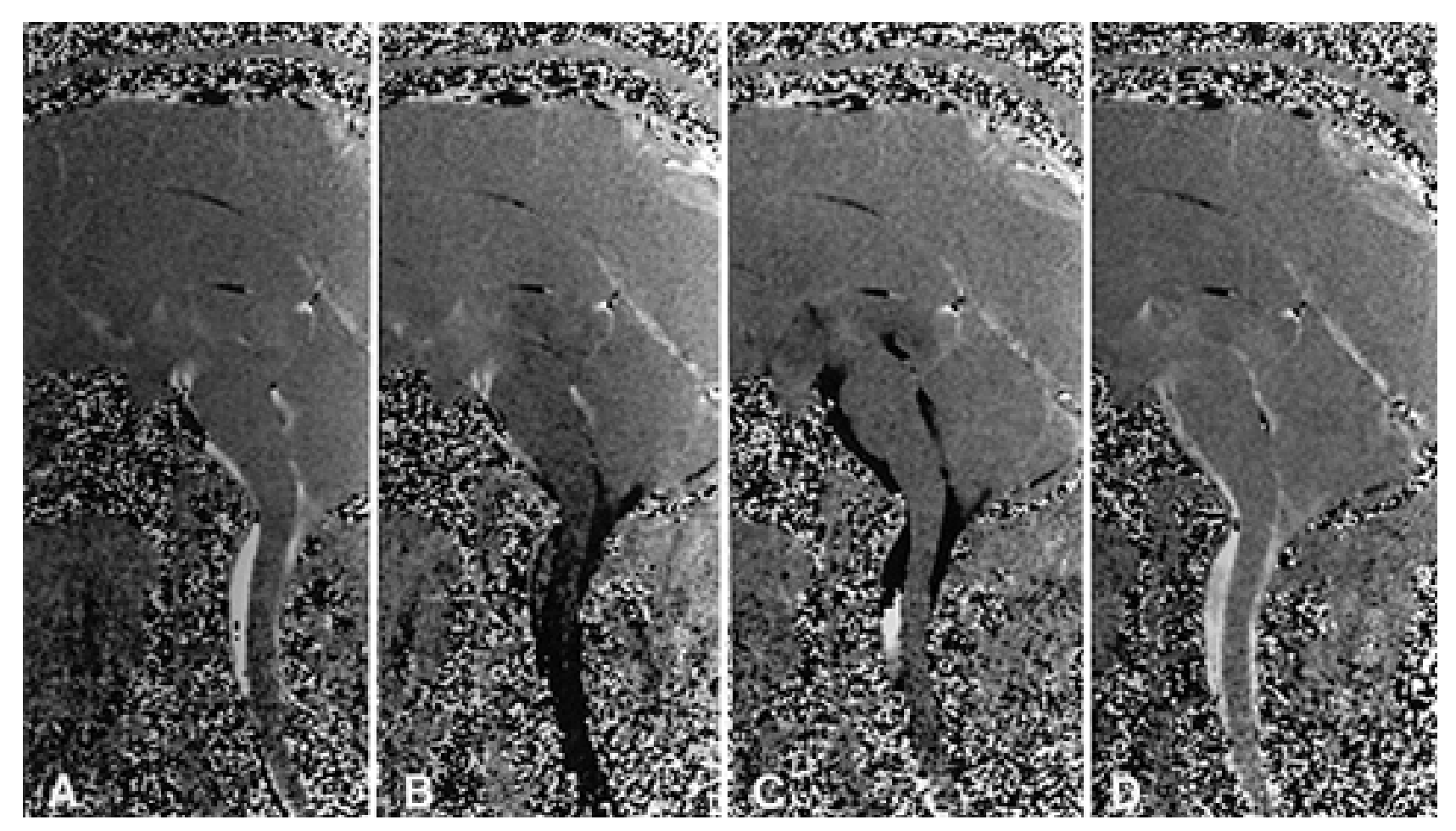

FIG. 6. Midsagittal phase-contrast images obtained in a patient 20 months after aqueductoplasty (same patient as shown in Figs. 3-5). A: Early during the cardiac cycle, there is a hyperintense signal in all CSF spaces indicating cranial flow (end of CSF diastole). B: Caudal flow (hypointense signal, CSF systole) started in the cervical subarachnoid space, whereas in the aqueduct there is still cranial flow. C: During CSF midsystole, there is caudal flow in all CSF spaces. D: During CSF diastole, there is cranial flow in all CSF spaces. See Video Clip 3.

The systolic temporal parameters are less variable than the diastolic parameters because variations in the R-R interval mainly influence the diastole. $7,24,39,42$ The peak CSF velocities (mean $4-5 \mathrm{~cm} / \mathrm{second}$ ), flow rates (mean $0.06 \mathrm{ml} /$ second), and stroke volumes (mean $26 \mu \mathrm{l}$ ) measured in our investigation compare favorably with results recently

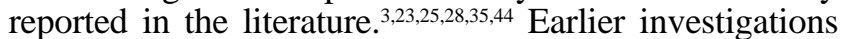
using in-plane measurements often found lower velocities. ${ }^{18,49}$ Because of the small size of the aqueduct relative to the sagittal slice thickness, accurate velocity calculations are difficult to obtain when quantifying in-plane phase-flow images. Partial volume effects contributed by static structures introduce severe phase diminution. This results in significant underestimation of CSF velocity. ${ }^{3,36}$ Nevertheless, even with the through-plane sequences and the use of high-resolution imaging units, there remains a considerable inaccuracy in the velocity data caused by nonlinearity of the gradients, eddy currents, partial volume effects, and placement of the ROI. ${ }^{3,28,33}$ The error is estimated to be approximately 10 to $15 \%$.,58 With very narrow aqueducts, the error may be even higher because noise and poor contrast make placement of the ROI difficult (unpublished data). Velocity measurements obtained in the basal cistern and cervical subarachnoid space are more reliable than velocity measurements obtained in smaller spaces such as the aqueduct.

As early as 1943 , O'Connell ${ }^{47}$ suggested that intracranial arterial pulsations are the main source of CSF pulsations. Bering ${ }^{5}$ proposed that choroid plexus pulsations are responsible for CSF motion. Du Boulay's ${ }^{13}$ cineventriculographic investigations seemed to confirm O'Connell's theory. He claimed that the third ventricle is the site of the CSF pump. Recent phase-contrast MR imaging studies of CSF flow confirm the concept of a cardiac-driven CSF pump. There is an expansion of the brain caused by arterial blood inflow during systole and relaxation of the brain caused by blood outflow during diastole..$^{10,24,49}$ These pulsatile changes in intracranial arterial blood volume shift the CSF. ${ }^{19}$ Furthermore, brain parenchyma motion contributes to CSF flow. ${ }^{16,19,20}$ Systolic downward movement of the brain has been deemed responsible for a piston action of the brain. ${ }^{24}$ Brainstem velocities of approximately $1.5 \mathrm{~mm} /$ second have been measured. ${ }^{16,20,26}$ The CSF and brain motions represent responses to vascular pulsations as the primary driving force. ${ }^{1}$

Retrospective cardiac gating has been proposed as the preferred triggering method for CSF flow quantification. ${ }^{6,7,46}$ This technique enables continuous measurement throughout the cardiac cycle. As the $\mathrm{R}$ wave is recorded, the data are sorted retrospectively. Advantages compared with prospective gating are use of the averaging approach to define the zero-velocity point and fewer shifts within the phase background caused by eddy currents. ${ }^{46}$ Nevertheless, we used prospective cardiac gating for flow quantification because, in our experience (unpublished data) and in the experience of others ${ }^{56}$ prospective gating has been less sensitive to disturbances such as irregular electrocardiographic signals. Furthermore, with prospec- 


\section{Cerebrospinal fluid flow after endoscopic aqueductoplasty}

tive cardiac gating, two acquisitions could be performed, which is not possible using our retrospective cardiac-gating technique. With the use of retrospective cardiac gating, the relative signal-to-noise ratio of 1 would decrease to 0.71 , which is particularly relevant in small anatomical structures such as the aqueduct. ${ }^{17}$ The partial volume effects would increase, because the placement of the ROI within the aqueduct would be less accurate. The main disadvantage of prospective cardiac gating is that the whole cardiac cycle is not covered and, hence, the diastolic flow cannot be completely evaluated. However, our intention was primarily to obtain as accurate quantitative CSF flow data as possible by using an acceptable imaging time.

Endoscopic third ventriculostomy has become the preferred treatment for hydrocephalus caused by aqueductal stenosis. ${ }^{29-31,57}$ The procedure is considered to be straightforward and safe. Although complications are rare, nearfatal and even fatal vascular injuries have been reported. ${ }^{27,45,51}$ Furthermore, sometimes the floor of the third ventricle is very tough, requiring considerable force to perforate it, which may traumatize surrounding structures such as the hypothalamus or pituitary stalk. Finally, the anatomy of the floor may be very distorted and lack landmarks, which makes orientation difficult. Therefore, endoscopic aqueductoplasty offers a reasonable alternative for the treatment of membranous or short aqueductal stenoses. Compared with third ventriculostomy, aqueductoplasty has some advantages: 1) aqueductoplasty restores the physiological CSF pathway; 2) aqueductoplasty carries no risk of major vessel injury; 3 ) arachnoid adhesions, which might interfere with CSF circulation, do not occur around the aqueduct but are occasionally found below the floor of the third ventricle; and 4) strictures of the aqueduct are easily perforated. In our opinion, an aqueductoplasty of a membranous stenosis is less traumatic than a third ventriculostomy.$^{50}$ However, endoscopic aqueductoplasty is indicated only in a select group of patients (16\% of our endoscopically treated patients with hydrocephalus).

\section{Conclusions}

The limitation of net CSF flow as well as pulsatile CSF flow through the aqueduct, as a cause of hydrocephalus, ${ }^{61}$ can effectively be treated using endoscopic aqueductoplasty.

The results of our investigation show that endoscopic aqueductoplasty restores the anatomical patency of the aqueduct and also seems to restore physiological CSF flow through the aqueduct. Therefore, we currently consider endoscopic aqueductoplasty to be the therapy of first choice in the treatment of membranous and short stenoses.

\section{Acknowledgments}

We thank Urte Menzel and Kathrin Fink for their assistance in performing the MR imaging examinations. We are indebted to Manuela Jahnke for the photographic work.

\section{References}

1. Alperin N, Vikingstad EM, Gomez-Anson B, et al: Hemodynamically independent analysis of cerebrospinal fluid and brain motion observed with dynamic phase contrast MRI. Magn Reson Med 35:741-754, 1996
2. Atlas SW, Mark AS, Fram EK: Aqueductal stenosis: evaluation with gradient-echo rapid MR imaging. Radiology 169: 449-453, 1988

3. Barkhof F, Kouwenhoven M, Scheltens P, et al: Phase-contrast cine MR imaging of normal aqueductal CSF flow. Effect of aging and relation to CSF void on modulus MR. Acta Radiol 35: 123-130, 1994

4. Bergstrand G, Bergström M, Nordell B, et al: Cardiac gated MR imaging of cerebrospinal fluid flow. J Comput Assist Tomogr 9:1003-1006, 1985

5. Bering EA Jr: Choroid plexus and arterial pulsation of cerebrospinal fluid. Demonstration of the choroid plexuses as a cerebrospinal fluid pump. Arch Neurol Psychiatry 73:165-173, 1955

6. Bhadelia RA, Bogdan AR, Kaplan RF, et al: Cerebrospinal fluid pulsation amplitude and its quantitative relationship to cerebral blood flow pulsations: a phase-contrast MR flow imaging study. Neuroradiology 39:258-264, 1997

7. Bhadelia RA, Bogdan AR, Wolpert SM: Analysis of cerebrospinal fluid flow waveforms with gated phase-contrast MR velocity measurements. AJNR 16:389-400, 1995

8. Bhadelia RA, Bogdan AR, Wolpert SM, et al: Cerebrospinal fluid flow waveforms: analysis in patients with Chiari I malformation by means of gated phase-contrast MR imaging velocity measurements. Radiology 196: 195-202, 1995

9. Bhadelia RA, Bogdan AR, Wolpert SM: Cerebrospinal fluid flow waveforms: effect of altered cranial venous outflow. A phase-contrast MR flow imaging study. Neuroradiology 40: 283-292, 1998

10. Bradley WG Jr, Kortman KE, Burgoyne B: Flowing cerebrospinal fluid in normal and hydrocephalic states: appearance on MR images. Radiology 159:611-616, 1986

11. Bradley WG Jr, Scalzo D, Queralt J, et al: Normal-pressure hydrocephalus: evaluation with cerebrospinal fluid flow measurements at MR imaging. Radiology 198:523-529, 1996

12. Chu D, Levin DN, Alperin N: Assessment of the biomechanical state of intracranial tissues by dynamic MRI of cerebrospinal fluid pulsations: a phantom study. Magn Reson Imaging 16: 1043-1048, 1998

13. du Boulay GH: Pulsatile movements in the CSF pathways. Br J Radiol 39:255-262, 1966

14. Egeler-Peerdeman SM, Barkhof F, Walchenbach R, et al: Cine phase-contrast MR imaging in normal pressure hydrocephalus patients: relation to surgical outcome. Acta Neurochir Suppl 71:340-342, 1998

15. Eguchi T, Taoka T, Nikaido Y, et al: Cine-magnetic resonance imaging evaluation of communication between middle cranial fossa arachnoid cysts and cisterns. Neurol Med Chir 36: 353-357, 1996

16. Enzmann DR: Brain motion: measurement with phase-contrast MR imaging. Radiology 185:653-660, 1992

17. Enzmann DR, Pelc NJ: Cerebrospinal fluid flow measured by phase-contrast cine MR. AJNR 14:1301-1307, 1993

18. Enzmann DR, Pelc NJ: Normal flow patterns of intracranial and spinal cerebrospinal fluid defined with phase-contrast cine MR imaging. Radiology 178:467-474, 1991

19. Feinberg DA: Functional magnetic resonance imaging. Application to degenerative brain disease and hydrocephalus. Neuroimag Clin North Am 5:125-134, 1995

20. Feinberg DA, Mark AS: Human brain motion and cerebrospinal fluid circulation demonstrated with MR velocity imaging. Radiology 163: 793-799, 1987

21. Fischbein NJ, Ciricillo SF, Barr RM, et al: Endoscopic third ventriculocisternostomy: MR assessment of patency with 2-D cine phase-contrast versus T2-weighted fast spin echo technique. Pediatr Neurosurg 28:70-78, 1998

22. Gideon P, Stahlberg F, Thomsen C, et al: Cerebrospinal fluid flow and production in patients with normal pressure hydrocephalus studied by MRI. Neuroradiology 36:210-215, 1994 
23. Gideon $\mathrm{P}$, Thomsen $\mathrm{C}$, Ståhlberg F, et al: Cerebrospinal fluid production and dynamics in normal aging: a MRI phase-mapping study. Acta Neurol Scand 89:362-366, 1994

24. Greitz D, Franck A, Nordell B: On the pulsatile nature of intracranial and spinal CSF-circulation demonstrated by MR imaging. Acta Radiol 34:321-328, 1993

25. Greitz D, Hannerz J, Rähn T, et al: MR imaging of cerebrospinal fluid dynamics in health and disease. On the vascular pathogenesis of communicating hydrocephalus and benign intracranial hypertension. Acta Radiol 35:204-211, 1994

26. Greitz D, Wirestam R, Franck A, et al: Pulsatile brain movement and associated hydrodynamics studied by magnetic resonance phase imaging. The Monro-Kellie doctrine revisited. Neuroradiology 34:370-380, 1992

27. Handler MH, Abbott R, Lee M: A near-fatal complication of endoscopic third ventriculostomy: case report. Neurosurgery 35:525-528, 1994

28. Henry-Feugeas MC, Idy-Peretti I, Blanchet B, et al: Temporal and spatial assessment of normal cerebrospinal fluid dynamics with MR imaging. Magn Reson Imaging 11:1107-1118, 1993

29. Hopf NJ, Grunert P, Fries G, et al: Endoscopic third ventriculostomy: outcome analysis of 100 consecutive procedures. Neurosurgery 44:795-806, 1999

30. Jones RFC, Brazier DH, Kwok BCT, et al: Neuroendoscopic third ventriculostomy, in Cohen A, Haines SJ (eds): Minimally Invasive Techniques in Neurosurgery. Baltimore: Williams \& Wilkins, 1995, pp 33-48

31. Jones RFC, Kwok BCT, Stening WA, et al: The current status of endoscopic third ventriculostomy in the management of noncommunicating hydrocephalus. Minim Invasive Neurosurg 37:28-36, 1994

32. Kadowaki C, Hara M, Numoto M, et al: Cine magnetic resonance imaging of aqueductal stenosis. Childs Nerv Syst 11: $107-111,1995$

33. Katayama S, Asari S, Ohmoto T: Quantitative measurement of normal and hydrocephalic cerebrospinal fluid flow using phase contrast cine MR imaging. Acta Med Okayama 47:157-168, 1993

34. Kim MH, Shin KM, Song JH: Cine MR CSF flow study in hydrocephalus: what are the valuable parameters? Acta Neurochir Suppl 71:343-346, 1998

35. Kolbitsch C, Schocke M, Lorenz IH, et al: Phase-contrast MRI measurement of systolic cerebrospinal fluid peak velocity $\left(\mathrm{CSFV}_{\text {Peak }}\right)$ in the aqueduct of Sylvius. Anesthesiology 90: 1546-1550, 1999

36. Kraft KA, Fei DY, Fatouros PP: Quantitative phase-velocity MR imaging of in-plane laminar flow: effect of fluid velocity, vessel diameter, and slice thickness. Med Phys 19:79-85, 1992

37. Kunz U, Goldmann A, Bader C, et al: Endoscopic fenestration of the 3rd ventricular floor in aqueductal stenosis. Minim Invasive Neurosurg 37:42-47, 1994

38. Lev S, Bhadelia RA, Estin D, et al: Functional analysis of third ventriculostomy patency with phase-contrast MRI velocity measurements. Neuroradiology 39:175-179, 1997

39. Levy LM, Di Chiro G: MR phase imaging and cerebrospinal fluid flow in the head and spine. Neuroradiology 32:399-406, 1990

40. Maeder P, Gudinchet F, Meuli R, et al: Dynamic MRI of cerebrospinal fluid flow in endoscopic percutaneous ventriculostomy. Br J Neurosurg 12:18-22, 1998

41. Maier SE, Hardy CJ, Jolesz FA: Brain and cerebrospinal fluid motion: real-time quantification with M-mode MR imaging. Radiology 193:477-483, 1994

42. Mark AS, Feinberg DA, Brant-Zawadzki MN: Changes in size and magnetic resonance signal intensity of the cerebral CSF spaces during the cardiac cycle as studied by gated, highresolution magnetic resonance imaging. Invest Radiol 22: 290-297, 1987
43. Mascalchi M, Ciraolo L, Tanfani G, et al: Cardiac-gated phase MR imaging of aqueductal CSF flow. J Comput Assist Tomogr 12:923-926, 1988

44. Mase M, Yamada K, Banno T, et al: Quantitative analysis of CSF flow dynamics using MRI in normal pressure hydrocephalus. Acta Neurochir Suppl 71:350-353, 1998

45. McLaughlin MR, Wahlig JB, Kaufmann AM, et al: Traumatic basilar aneurysm after endoscopic third ventriculostomy: case report. Neurosurgery 41:1400-1404, 1997

46. Nitz WR, Bradley WG Jr, Watanabe AS, et al: Flow dynamics of cerebrospinal fluid: assessment with phase-contrast velocity MR imaging performed with retrospective cardiac gating. Radiology 183:395-405, 1992

47. O'Connell JEA: The vascular factor in intracranial pressure and the maintenance of the cerebrospinal fluid circulation. Brain 66:204-228, 1943

48. Oka K, Go Y, Kin Y, et al: The radiographic restoration of the ventricular system after third ventriculostomy. Minim Invasive Neurosurg 38:158-162, 1995

49. Quencer RM, Donovan Post MJ, Hinks RS: Cine MR in the evaluation of normal and abnormal CSF flow: intracranial and intraspinal studies. Neuroradiology 32:371-391, 1990

50. Schroeder HWS, Gaab MR: Endoscopic aqueductoplasty: technique and results. Neurosurgery 45:508-518, 1999

51. Schroeder HWS, Warzok RW, Assaf JA, et al: Fatal subarachnoid hemorrhage after endoscopic third ventriculostomy. Case report. J Neurosurg 90:153-155, 1999

52. Schroth G, Klose U: Cerebrospinal fluid flow. II. Physiology of respiration-related pulsations. Neuroradiology 35:10-15, 1992

53. Sherman JL, Citrin CM: Magnetic resonance demonstration of normal CSF flow. AJNR 7:3-6, 1986

54. Sherman JL, Citrin CM, Bowen BJ, et al: MR demonstration of altered cerebrospinal fluid flow by obstructive lesions. AJNR 7:571-579, 1986

55. Sherman JL, Citrin CM, Gangarosa RE, et al: The MR appearance of CSF flow in patients with ventriculomegaly. AJR 148: 193-199, 1987

56. Stoeter P, Brühl K, Gawehn J: MR-Diagnostik von Liquorfluss und Hirnbewegung. Extracta Psychiatr 12:17-24, 1998

57. Teo C: Third ventriculostomy in the treatment of hydrocephalus: experience with more than 120 cases, in Hellwig D, Bauer BL (eds): Minimally Invasive Techniques for Neurosurgery: Current Status and Future Perspectives. Berlin: SpringerVerlag, 1998, pp 73-76

58. Tintera J, Brühl K, Stoeter P: Systematic errors by CSF flow quantification using phase-contrast (PC) MRI. Neuroradiology 38 (Suppl 2):83-84, 1996 (Abstract)

59. van den Hout JHW, Bakker CJG, Mali WPTM, et al: Magnetic resonance imaging of the cerebral aqueduct. Signal intensity time curves demonstrated by fast acquisition with multiple excitation (FAME). Invest Radiol 24:855-860, 1989

60. Watabe N, Tominaga T, Shimizu H, et al: Quantitative analysis of cerebrospinal fluid flow in patients with cervical spondylosis using cine phase-contrast magnetic resonance imaging. Neurosurgery 44:779-784, 1999

61. White DN, Wilson KC, Curry GR, et al: The limitation of pulsatile flow through the aqueduct of Sylvius as a cause of hydrocephalus. J Neurol Sci 42:11-51, 1979

62. Wilcock DJ, Jaspan T, Worthington BS, et al: Neuro-endoscopic third ventriculostomy: evaluation with magnetic resonance imaging. Clin Radiol 52:50-54, 1997

Manuscript received October 7, 1999.

Accepted in final form April 17, 2000.

Address reprint requests to: Henry W. S. Schroeder, M.D., Department of Neurosurgery, Ernst Moritz Arndt University, Sauerbruchstrasse, D-17487 Greifswald, Germany. email: schroedh@ mail.uni-greifswald.de. 